\title{
Glomerular IgG deposition predicts renal outcome in patients with IgA nephropathy
}

Dong Ho Shin ${ }^{1}$, Beom Jin $\mathrm{Lim}^{2}$, In Mi Han ${ }^{3}$, Seung Gyu Han ${ }^{3}$, Young Eun Kwon ${ }^{3}$, Kyoung Sook Park ${ }^{3}$, Mi Jung Lee ${ }^{3}$, Hyung Jung Oh ${ }^{3}$, Jung Tak Park ${ }^{3}$, Seung Hyeok Han ${ }^{3}$, Shin-Wook Kang ${ }^{3}$ and Tae-Hyun Yoo $^{3}$

${ }^{1}$ Department of Internal Medicine, Kangdong Sacred Heart Hospital, Hallym University, Seoul, Korea;

${ }^{2}$ Department of Pathology, Yonsei University College of Medicine, Seoul, Korea and ${ }^{3}$ Department of Internal Medicine, Yonsei University College of Medicine, Seoul, Korea

Glomerular IgG deposition is frequently observed in patients with IgA nephropathy. However, the association between glomerular IgG deposition and progression of IgA nephropathy is uncertain. Six hundred and twentyseven patients with biopsy-proven IgA nephropathy were recruited. Histological variables of the Oxford classification (Oxford-MEST) and the presence of glomerular IgG deposits were assessed. Renal progression defined as end-stage renal disease or $\mathbf{5 0 \%}$ reduction in estimated glomerular filtration rate was analyzed using Kaplan-Meier methods and Cox regression analysis. Of the study population, 200 patients $(31.9 \%)$ had glomerular IgG deposition on immunofluorescence staining. During a mean follow-up of $56.8 \pm 37.5$ months, the rate of renal progression was significantly higher in the IgA nephropathy patients with glomerular IgG deposition compared with the IgA nephropathy patients without glomerular IgG deposition (39.8 vs 12.3 per 1000 patientyears; $P<0.001)$. Of patients with IgG deposition, $178(28.3 \%), 20(3.2 \%)$, and $2(0.3 \%)$ patients had mild, moderate, and marked glomerular IgG deposits, receptively. Kaplan-Meier analysis revealed that cumulative renal survival was significantly lower in IgA nephropathy patients with the higher intensity of glomerular IgG deposits $(\boldsymbol{P}<\mathbf{0 . 0 0 1})$. In addition, Cox regression analysis revealed that moderate and marked glomerular IgG deposits significantly predicted renal outcome independent of Oxford-MEST and clinical variables (HR, 2.97; 95\% $\mathrm{Cl}, 1.01-8.77 ; P=0.04)$. This study showed that that glomerular IgG deposition was independently associated with poor renal outcome in patient with IgA nephropathy.

Modern Pathology (2016) 29, 743-752; doi:10.1038/modpathol.2016.77; published online 22 April 2016

IgA nephropathy is the most common cause of primary glomerulonephritis worldwide. ${ }^{1}$ It is characterized by predominant IgA deposition in the glomerular mesangium. Although the clinical presentation and course of IgA nephropathy is quite variable, ranging from asymptomatic microscopic hematuria to rapidly progressive glomerulonephritis, IgA nephropathy progresses insidiously and 10-30\% of patients reach end-stage renal disease within 20 years. ${ }^{2-7}$ Recent studies have revealed that the pathogenesis of IgA nephropathy involves an autoimmune response with the formation of abnormally glycosylated IgA1. Lymphocytes from patients with IgA nephropathy produce IgG that form immune

Correspondence: BJ Lim, MD, PhD, Department of Pathology, Yonsei University College of Medicine, 211 Eonju-ro, Gangnamgu, Seoul 135-720, Korea or Professor T-H Yoo, MD, PhD, Department of Internal Medicine, Yonsei University College of Medicine, 50-1 Yonsei-ro, Seodaemun-gu, Seoul 120-752, Korea. E-mail: bjlim@yuhs.ac or yoosy0316@yuhs.ac

Received 27 October 2015; revised 22 March 2016; accepted 24 March 2016; published online 22 April 2016 complexes with abnormally glycosylated IgA1. Binding of these immune complexes to mesangial cells induces mesangial damage that leads to renal injury. ${ }^{8}$ Therefore, this pathogenetic mechanism underlying IgA nephropathy suggests a potential clinical involvement of IgG autoantibodies in IgA nephropathy patients. Suzuki et $a l^{9}$ also described how specific IgG autoantibodies respond to the abnormally glycosylated IgA1. A diagnostic assay for detection of IgA1-IgG is currently unavailable; however, clinical mesangial IgG deposition under kidney biopsy may provide indirect evidence of the immune reaction involving IgA1.

Immunofluorescence findings showed that IgA alone is deposited in $<25 \%$ and other immunoglobulins usually accompany IgA, including IgG and IgM, respectively. ${ }^{10}$ Interestingly, there are some reports showing the prognostic role of mesangial IgG deposition in IgA nephropathy. Animal models for IgA nephropathy have demonstrated that mesangial co-deposition of IgA and IgG aggravated glomerular inflammation and proteinuria. ${ }^{11}$ In early IgA 
nephropathy, mesangial IgG deposition was associated with the development of hypertension and decreased renal function. ${ }^{12}$ Moreover, recent observational studies have suggested that mesangial IgG deposition in IgA nephropathy could be associated with disease severity and renal progression. ${ }^{13,14}$ The Oxford-MEST classification of IgA nephropathy identifies four types of lesions as specific pathological features that may act as independent predictors of clinical outcomes: mesangial hypercellularity (M), endocapillary hypercellularity (E), segmental glomerulosclerosis (S), and tubular atrophy/interstitial fibrosis (T). ${ }^{15,16}$ However, this new classification does not include immunofluorescence patterns, which might reflect the pathophysiological mechanisms active in IgA nephropathy. Our aim was to investigate the prognostic significance of glomerular IgG deposition in patients with IgA nephropathy; thus in the present study, we incorporated glomerular IgG deposits into the Oxford-MEST classification and investigated its prognostic value.

\section{Materials and methods}

\section{Ethics Statement}

This study was carried out in accordance with the Declaration of Helsinki and approved by the Institutional Review Board of Yonsei University Health System Clinical Trial Center. As the current study was a retrospective study and the study subjects were de-identified, the Institutional Review Board waived the need for written consent from the patients.

\section{Study Subjects}

From January 2000 to December 2013, 763 patients were diagnosed with IgA nephropathy via renal biopsy at a large academic center in Korea. Patients with Henoch-Schonlein purpura were considered ineligible. Cases with an initial estimated glomerular filtration $<30 \mathrm{ml} / \mathrm{min}$ per $1.73 \mathrm{~m}^{2}$ were excluded $(n=30)$. We also excluded patients who had features of the secondary cause of mesangial IgA deposition, such as IgA-dominant acute postinfectious glomerulonephritis and systemic lupus erythematosus $(n=24)$. In addition, patients aged $<20$ years $(n=10)$ or $>75$ years $(n=5)$, those with inadequate biopsy samples having a number of glomeruli $\leq 7$ $(n=15)$ and patients who initially presented with advanced chronic liver disease $(n=11)$ were also excluded. Finally, patients who were followed up for $<6$ months were excluded $(n=41)$. Overall, a total of 627 patients were included in this study.

\section{Data Collection}

At the time of the renal biopsy, demographic and clinical data such as age, sex, blood pressure, and combined medications were collected. Mean arterial pressure was defined as diastolic pressure plus a third of the pulse pressure. The concurrent laboratory tests, including urinary protein-to-creatinine ratio and serum creatinine, were measured from urine and blood samples. The estimated glomerular filtration was calculated using the CKD-EPI equation. ${ }^{17}$ During the follow-up period, patients who received corticosteroids, other immunosuppressants, or anti-hypertensive drugs were defined as those treated for $>6$ months.

\section{Renal Biopsy}

Originally, each sample was routinely processed for light microscopy, immunofluorescence microscopy, and electron microscopy. Light microscopic examination was performed with paraffin-embedded tissue under hematoxyliln-eosin, periodic acid-Schiff, aldehyde fuchsin orange $G$, and periodic acid-silver methenamine stains. For immunofluorescence microscopic examination, snap-frozen $3-\mu$ m-thick sections were used. Each section was reacted with antibodies against IgG, IgA, IgM, C1q, C3, C4, and fibrinogen (1:20, DAKOCytomation, Glostrup, Denmark). The degree of deposits was represented semiquantitatively (grade 0 , no or trace; grade 1, mild; grade 2, moderate; grade 3 , marked) in the mesangium and along the glomerular capillary loops, respectively. Finally, each item was represented by absence (score 0 ) and presence (scores 1-3). Of note, the method of immunofluorescence was basically the same throughout the research period. All renal biopsy specimens were re-assessed by a single pathologist according to the Oxford classification. ${ }^{15}$ The mesangial cellularity was scored 0 ( $<4$ mesangial cells/mesangial area), 1 (4-5 cells), 2 (6-7 cells), and 3 (>8 cells) for each glomerulus. The mean score of all glomeruli was classified as M0 $(\leq 0.5)$ and M1 $(>0.5)$. When more than half of glomeruli show score $\geq 1$, the case was classified as M1 without scoring each glomerulus. Segmental glomerulosclerosis and endocapillary hypercellularity were categorized as either present (S1 and E1) or absent (S0 and E0). Tubular atrophy/ interstitial fibrosis was classified as T0 $(0-25 \%$ of cortical area), T1 (26-50\% of cortical area), or T2 ( $>50 \%$ of cortical area).

\section{Study Outcomes}

The study end point was the onset of end-stage renal disease and $50 \%$ reduction in estimated glomerular filtration. End-stage renal disease was defined as initiation of renal replacement therapy, including permanent hemodialysis, peritoneal dialysis, or renal transplantation.

\section{Statistical Analysis}

Statistical analysis was performed using the SPSS software (version 20.0, IBM SPSS) or Stata software 
(version 11.0, StataCorp). The Kolmogorov-Smirnov test was used to analyze the normality of the distribution of parameters in continuous variables. Data that did not show a normal distribution were expressed as median and interquartile range. Categorical variables were expressed as number (percentage). To test for difference in categorical or continuous variables among group, chi-squared test, Mann-Whitney test, or Kruskal-Wallis test were used. The slope of the decline in renal function over time was calculated by linear regression analysis of serial estimated glomerular filtration for each patient. To determine an independent association between histopathological features and the decline in rate of renal function, univariate and multivariate linear regression analyses were used. Renal survival curves were generated with the Kaplan-Meier method, and between-group survival was compared by using the log-rank test. The independent prognostic values of clinical and pathological parameters for the study outcomes were analyzed by performing multiple Cox regression analysis. Harrell's $C$ index of each model was calculated to investigate the discriminatory ability. Using this index, we determined whether the degree of glomerular IgG deposits had additive value to Oxford-MEST lesions in the prediction of renal survival. All probabilities were two-tailed and the level of significance was set at 0.05 .

\section{Results}

\section{Baseline Characteristics According to the Degree of Glomerular IgG Deposits at the Time of Biopsy}

Patient demographic, clinical, and biochemical characteristics at the time of biopsy are shown in Table 1 . The median age was $32(25-42)$ years, and $302(48.2 \%)$ were male. The median mean arterial pressure and estimated glomerular filtration rate were $93.3(84.8-100.0)$ and $84.1(66.3-101.1) \mathrm{ml} /$ $\min / 1.73 \mathrm{~m}^{2}$. Gross hematuria was noted in $25.4 \%$ of patients. The median urinary protein-to-creatinine ratio was $0.6(0.1-1.6) \mathrm{g} / \mathrm{g}$. Of the 627 patients, 200 $(32 \%)$ patients had glomerular IgG deposition and $178(28.3 \%), 20(3.2 \%)$, and $2(0.3 \%)$ patients had mild, moderate, and marked glomerular IgG deposits, respectively. The subtypes of IgG were checked in 22 patients using archived frozen kidney tissues. Of these patients, only eight patients showed reliable results (Supplementary Table S1). Urinary proteinto-creatinine ratio $(0.5(0.0-1.3), 0.9(0.0-1.8), 1.6$ (0.6-3.6); $\quad P<0.001)$ was higher and estimated glomerular filtration (86.0 (67.9-102.1), 80.2 (61.9100.2), $79.8(63.8-89.0 ; P=0.02)]$ was lower as the degree of glomerular IgG deposits increased. Moreover, the proportion of T1 and T2 and the proportion of glomerular C3 deposits were higher as the degree of glomerular IgG deposits increased. In contrast, the mean age, sex, the proportion of patients with hypertension, systolic and diastolic blood pressure, serum cholesterol levels, triglyceride levels, estimated glomerular filtration, IgG, IgA, IgM, and C3 and $\mathrm{C} 4$ concentrations were comparable among the three groups.

\section{Comparison of Clinical Parameters at the Time of Kidney Biopsy and Decline Rate of Renal Function According to the Oxford Classification and Glomerular IgG Deposits}

The baseline distribution of the pathological variables and the comparison of clinical data according to the Oxford-MEST classification and the degree of glomerular IgG deposits are reported in Table 2 . At the time of biopsy, patients with a higher score for each MEST lesion on the Oxford classification and with the higher intensity of glomerular IgG deposits had greater proteinuria. Furthermore, baseline estimated glomerular filtration was significantly lower in patients with M1, E1, and T1/T2. The rate of decline in estimated glomerular filtration was greater in patients with $\mathrm{M} 1, \mathrm{~S} 1$, and $\mathrm{T} 1 / \mathrm{T} 2$ than in patients with M0, S0, and T0, respectively. In addition, the decline in estimated glomerular filtration was also greater as the degree of glomerular IgG deposits increased. In addition, a multivariate linear regression analysis was performed to identify pathological variables associated with a decline in kidney function. After adjustment for clinical variables, segmental sclerosis $(\beta=-0.16, P<0.001)$, tubular atrophy/interstitial fibrosis $(\beta=-0.19$, $P<0.001$ ), and the higher intensity of glomerular IgG deposits significantly correlated with the rate of estimated glomerular filtration decline $(\beta=-0.16$, $P<0.001$ ) (Table 2). However, the location of glomerular IgG deposits did not affect clinical parameters and renal outcome among patients with detectable glomerular IgG deposits (Supplementary Tables S2 and S3). Of note, there were no differences in urinary protein-to-creatinine ratio, estimated glomerular filtration, and the rate of decline in estimated glomerular filtration according to the any other glomerular immunoreactant deposits (Table 3).

\section{Prognostic Value of Glomerular IgG Deposition for Renal Outcome}

During a mean follow-up of $56.8 \pm 37.5$ months, the end point of $50 \%$ decline in estimated glomerular filtration was reached in $62(9.9 \%)$ patients and endstage renal disease was reached in $47(7.5 \%)$. None of the patients progressed to end-stage renal disease before reaching a 50\% decline in end-stage renal disease. A 50\% decrease in end-stage renal disease occurred more often in IgA nephropathy patients with glomerular IgG deposition (37 patients; 18.5\%) than in those without any detectable deposits (25 patients; $5.9 \%$ ), resulting in rates of 39.8 and 
Table 1 Baseline characteristics of the subjects according to the degree of glomerular IgG deposits at the time of biopsy

\begin{tabular}{|c|c|c|c|c|c|}
\hline \multirow{2}{*}{ Variables } & \multirow{2}{*}{ All $(\mathrm{N}=627)$} & \multicolumn{4}{|c|}{ Glomerular IgG deposition } \\
\hline & & $\begin{array}{l}\text { Grade } 0 \\
(\mathrm{~N}=427)\end{array}$ & $\begin{array}{l}\text { Grade } 1 \\
(\mathrm{~N}=178)\end{array}$ & $\begin{array}{l}\text { Grades } 2+3 \\
(\mathrm{~N}=22)\end{array}$ & $\begin{array}{l}\text { P-value for } \\
\text { trend }\end{array}$ \\
\hline Age & $32(25-42)$ & $34(25-41)$ & $33.0(25-42)$ & $30.5(27-35)$ & 0.46 \\
\hline Male (\%) & $302(48.2)$ & $205(48.0)$ & $89(50.0)$ & $8(36.4)$ & 0.48 \\
\hline Hypertension (\%) & $91(14.5)$ & $60(14.1)$ & $25(14.0)$ & $6(27.3)$ & 0.22 \\
\hline Systolic blood pressure (mm Hg) & $120(110-130)$ & $120(110-130)$ & $120(110-135)$ & $129(120-140)$ & 0.29 \\
\hline Diastolic blood pressure (mm Hg) & $80(70-85)$ & $80(70-83)$ & $80(70-87)$ & $80(70-85)$ & 0.44 \\
\hline Mean arterial pressure (mm Hg) & $93.3(84.8-100.0)$ & $93.3(84.5-100.0)$ & $93.3(84.0-100.0)$ & $93.3(90.0-101.7)$ & 0.38 \\
\hline Gross hematuria (\%) & $159(25.4)$ & $107(25.1)$ & $48(27.0)$ & $4(18.2)$ & 0.65 \\
\hline Urinary protein-to-creatinine ratio (g/g) & $0.6(0.1-1.6)$ & $0.5(0.0-1.3)$ & $0.9(0.0-1.8)$ & $1.6(0.6-3.6)$ & $<0.001$ \\
\hline Cholesterol (mg/dl) & $176(150-203)$ & $176(149-200)$ & $174(153-204)$ & $161.0(124-212)$ & 0.37 \\
\hline Triglycerides (mg/dl) & $115(81-166)$ & $114(76-167)$ & $121(96-165)$ & $124(91-168)$ & 0.13 \\
\hline $\begin{array}{l}\text { Estimated glomerular filtration rate } \\
\left(\mathrm{ml} / \mathrm{min} \text { per } 1.73 \mathrm{~m}^{2}\right)\end{array}$ & $84.1(66.3-101.1)$ & $86.0(67.9-102.3)$ & $80.2(61.9-100.2)$ & $79.8(63.8-89.0)$ & 0.02 \\
\hline $\begin{array}{l}30 \leq \text { estimated glomerular filtration } \\
\text { rate }<60, n(\%)\end{array}$ & $113(18.0)$ & $66(15.5)$ & $41(23.0)$ & $6(27.3)$ & 0.01 \\
\hline $\begin{array}{l}\text { Estimated glomerular filtration rate } \\
\geq 60, n(\%)\end{array}$ & $514(82.0)$ & $361(84.5)$ & $137(77.0)$ & $18(72.7)$ & \\
\hline Follow-up (months) & $48.0(28.7-76.7)$ & $48.0(29.8-76.7)$ & $49.2(27.6-79.2)$ & $43.3(18.7-69.4)$ & 0.46 \\
\hline Time to renal biopsy (months) & $13.2(8.9-19.1)$ & $14.3(8.6-21.2)$ & $12.1(9.9-18.1)$ & $19.5(18.2-24.3)$ & 0.01 \\
\hline IgG (mg/dl) & $1270(1100-1305)$ & 1275 (1110-1315) & $1280(1100-1315)$ & $1260(951-1300)$ & 0.16 \\
\hline $\operatorname{IgA}(\mathrm{mg} / \mathrm{dl})$ & $323(247-365)$ & $322(247-367)$ & 314 (247-365) & 365 (299-413) & 0.16 \\
\hline $\operatorname{IgM}(\mathrm{mg} / \mathrm{dl})$ & $125(98-156)$ & $125(96-156)$ & $134(101-166)$ & $125(99.2-169.0)$ & 0.25 \\
\hline C3 (mg/dl) & $96.7(95.4-108.0)$ & 96.7 (95.4-108.0) & $96.7(95.8-103.0)$ & $96.7(95.8-99.8)$ & 0.60 \\
\hline C4 (mg/dl) & $22.6(19.6-26.1)$ & $22.6(19.7-25.8)$ & $22.6(19.4-27.7)$ & $22.5(21.9-23.9)$ & 0.61 \\
\hline \multicolumn{6}{|l|}{ Treatment (\%) } \\
\hline Renin-angiotensin system blockade & $542(86.4)$ & 357 (83.6) & $167(93.8)$ & $18(80.0)$ & 0.01 \\
\hline Corticosteroid & $42(6.7)$ & $22(5.2)$ & $19(10.7)$ & $1(4.5)$ & 0.04 \\
\hline Other immunosuppressants & $8(1.3)$ & $4(0.9)$ & $4(2.2)$ & $0(0.0)$ & 0.37 \\
\hline \multicolumn{6}{|l|}{ Pathological variables (\%) } \\
\hline \multicolumn{6}{|l|}{ Oxford classification } \\
\hline M1 & $286(45.6)$ & $188(45.3)$ & $85(50.9)$ & $13(61.9)$ & 0.19 \\
\hline S1 & $288(45.9)$ & 196 (45.9) & $84(47.2)$ & $8(36.4)$ & 0.63 \\
\hline E1 & $35(5.6)$ & $22(5.2)$ & $11(6.2)$ & $2(9.1)$ & 0.68 \\
\hline T1 & $108(17.2)$ & $59(13.8)$ & $41(23.0)$ & $8(36.4)$ & $<0.001$ \\
\hline $\mathrm{T} 2$ & $35(5.6)$ & $11(2.6)$ & $22(12.4)$ & $2(9.1)$ & \\
\hline \multicolumn{6}{|l|}{ Immunofluorescence } \\
\hline $\operatorname{IgM}$ & $193(31)$ & $123(29.1)$ & $64(36.2)$ & $6(27.3)$ & 0.22 \\
\hline $\mathrm{C} 1 \mathrm{q}$ & $33(5.3)$ & $13(3.1)$ & $16(9.0)$ & $4(18.2)$ & 0.001 \\
\hline $\mathrm{C} 3$ & $531(85.2)$ & 338 (79.9) & $171(96.1)$ & $22(100.0)$ & $<0.001$ \\
\hline $\mathrm{C} 4$ & $27(4.3)$ & $17(4.0)$ & $7(3.9)$ & 3 (13.6) & 0.09 \\
\hline
\end{tabular}

Abbreviations: E1, presence of endocapillary hypercellularity; M1, mesangial hypercellularity >0.5; G grade 0, no or trace IgG deposits; G grade 1, mild IgG deposits; G grade 2, moderate IgG deposits; G grade 3, marked IgG deposits; S1, presence of segmental glomerulosclerosis; T1, tubular atrophy/interstitial fibrosis $26-50 \%$ of cortical area; T2, tubular atrophy/interstitial fibrosis $>50 \%$ of cortical area.

Note: Values are expressed as median (interquartile range) or number (percentage).

Calculation of mean arterial pressure, estimated glomerular filtration rate,and proteinuria is detailed in the text.

12.3 per 1000 patient-years, respectively $(P<0.001)$. The incidence of end-stage renal disease was also significantly higher in the IgA nephropathy patients with glomerular IgG deposition relative to the IgA nephropathy patients without glomerular IgG deposition (28.0 vs 10.3 per 1000 patient-years; $P=0.001$ ). The odds ratios for halving of estimated glomerular filtration and achieving end-stage renal disease in the IgA nephropathy patients with glomerular IgG deposition were 3.7 (95\% confidence interval (CI), 2.1-6.3; $P<0.001)$ and 2.9 (95\% CI, 1.6-5.3; $P=0.001$ ), respectively (Table 4). KaplanMeier analysis revealed that cumulative renal survival was significantly lower in IgA nephropathy patients with the higher intensity of glomerular IgG deposits (Figure $1, P<0.001$ ).

In a multivariate Cox proportional model, renal outcome for end-stage renal disease and $50 \%$ reduction in estimated glomerular filtration was significantly associated with urinary protein-to-creatinine ratio and estimated glomerular filtration (Table 5, model 1). When the degree of glomerular IgG deposits was included in a multivariate model, the higher intensity of glomerular IgG deposits remained independent predictors of renal outcomes (model 2). In addition, some of the components in the Oxford-MEST lesion classification were independent predictors of adverse renal outcomes in a 
Table 2 Comparison of clinical parameters at the time of kidney biopsy and decline rate of renal function according to the Oxford classification and glomerular IgG deposits

\begin{tabular}{|c|c|c|c|c|c|}
\hline & \multicolumn{3}{|c|}{ At the time of biopsy } & \multicolumn{2}{|c|}{$\begin{array}{l}\text { Rate of renal function decline } \\
\text { (linear regression) }\end{array}$} \\
\hline & $\begin{array}{c}\text { Mean arterial pressure } \\
\text { (mm Hg) }\end{array}$ & $\begin{array}{l}\text { Urinary protein-to- } \\
\text { creatinine ratio }\end{array}$ & $\begin{array}{l}\text { Estimated glomerular filtration } \\
\text { rate }\left(\mathrm{ml} / \mathrm{min} \text { per } 1.73 \mathrm{~m}^{2}\right)\end{array}$ & $\begin{array}{l}\text { Univariate (ml/min per } \\
1.73 \mathrm{~m}^{2} \text { per year) }\end{array}$ & $\begin{array}{l}\text { Mutivariate } \beta \\
\text { (s.d. })^{\mathrm{a}}\end{array}$ \\
\hline M0 & $93.3(83.3-100.7)$ & $0.6(0.0-1.3)$ & 89.1 (73.0-104.9) & $0.4(0.2-1.0)$ & \\
\hline M1 & $93.3(84.7-100.0)$ & $0.7(0.0-1.9)$ & $82.8(62.9-96.9)$ & $0.5(0.2-1.3)$ & $-0.07(0.34)$ \\
\hline$P$-value & 0.42 & 0.04 & $<0.001$ & 0.03 & 0.11 \\
\hline E0 & 92.7 (83.3-100.8) & $0.6(0.0-1.4)$ & 85.7 (66.5-102.5) & $0.5(0.2-1.1)$ & \\
\hline E1 & $93.3(85.0-100.0)$ & $1.9(0.2-3.4)$ & $75.6(61.3-89.0)$ & $0.7(0.3-2.4)$ & $-0.05(0.72)$ \\
\hline$P$-value & 0.53 & $<0.001$ & 0.01 & 0.13 & 0.24 \\
\hline So & $93.3(83.3-100.0)$ & $0.1(0.0-0.8)$ & 86.8 (68.4-102.5) & $0.3(0.1-0.6)$ & \\
\hline S1 & $93.3(86.5-100.0)$ & $1.2(0.4-1.9)$ & $84.1(64.3-101.2)$ & $0.8(0.5-1.8)$ & $-0.16(0.35)$ \\
\hline$P$-value & 0.26 & $<0.001$ & 0.7 & $<0.001$ & $<0.001$ \\
\hline T0 & $93.3(84.5-100.0)$ & $0.6(0.0-1.4)$ & 89.1 (70.7-103.5) & $0.4(0.2-1.0)$ & \\
\hline $\mathrm{T} 1$ & $93.3(85.8-100.0)$ & $0.7(0.0-2.1)$ & $78.2(62.1-94.2)$ & $0.6(0.3-1.2)$ & \\
\hline $\mathrm{T} 2$ & 96.7 (86.0-101.8) & $1.3(0.1-3.7)$ & $42.3(36.5-56.7)$ & $8.0(1.2-10.8)$ & $-0.19(1.60)$ \\
\hline$P$-value & 0.71 & $<0.001$ & $<0.001$ & $<0.001$ & $<0.001$ \\
\hline $\mathrm{G}$ grade 0 & $93.3(84.5-100.0)$ & $0.5(0.0-1.3)$ & $86.0(67.9-102.1)$ & $0.4(0.2-0.9)$ & \\
\hline G grade 1 & $93.3(84.0-100.0)$ & $0.9(0.0-1.8)$ & $80.2(62.3-100.0)$ & $0.6(0.3-2.3)$ & \\
\hline$G$ grade $2+3$ & $93.3(90.0-101.7)$ & $1.6(0.6-3.6)$ & $79.8(63.8-89.0)$ & $2.3(0.6-6.7)$ & $-0.16(0.33)$ \\
\hline$P$-value & 0.38 & $<0.001$ & 0.02 & $<0.001$ & $<0.001$ \\
\hline
\end{tabular}

Abbreviations: E0, absence of endocapillary; E1, presence of endocapillary hypercellularity; G grade 0, no or trace IgG deposits; G grade 1, mild IgG deposits; G grade 2, moderate IgG deposits; G grade 3, marked IgG deposits; M0, mesangial hypercellularity score of $\leq 0.5$; M1, mesangial hypercellularity score $>0.5$; S0, absence of segmental glomerulosclerosis; S1, presence of segmental glomerulosclerosis; T0, tubular atrophy/ interstitial fibrosis $\leq 25 \%$ of cortical area; T1, tubular atrophy/interstitial fibrosis $26-50 \%$ of cortical area; T2, tubular atrophy/interstitial fibrosis $>50 \%$ of cortical area.

Note: Values are expressed as median (interquartile range).

Calculation of mean arterial pressure, estimated glomerular filtration rate, and proteinuria is detailed in the text.

${ }^{\mathrm{a}}$ Mutivariate models are adjusted for initial estimated glomerular filtration rate, mean arterial pressure, and proteinuria.

Table 3 Comparison of clinical parameters at the time of kidney biopsy and decline rate of renal function according to the other immunofluorescence deposits

At the time of biopsy

\begin{tabular}{|c|c|c|}
\hline $\begin{array}{c}\text { Mean arterial pressure } \\
\text { (mm Hg) }\end{array}$ & $\begin{array}{l}\text { Urinary protein-to- } \\
\text { creatinine ratio }\end{array}$ & $\begin{array}{l}\text { Estimated glomerular filtration } \\
\text { rate }\left(\mathrm{ml} / \mathrm{min} \text { per } 1.73 \mathrm{~m}^{2}\right)\end{array}$ \\
\hline
\end{tabular}

\begin{tabular}{lcl}
\hline IgM (-) & $93.3(84.5-99.0)$ & $0.5(0.0-1.4)$ \\
IgM (+) & $93.3(86.3-103.3)$ & $0.7(0.0-1.7)$ \\
$P$-value & 0.01 & 0.06 \\
C1q (-) & $93.3(84.8-100.0)$ & $0.6(0.0-1.5)$ \\
C1q (+) & $96.0(88.3-100.0)$ & $1.1(0.1-2.1)$ \\
$P$-value & 0.50 & 0.09 \\
C3 (-) & $93.3(86.7-103.0)$ & $0.9(0.0-1.7)$ \\
C3 (+) & $93.3(84.2-100.0)$ & $0.5(0.0-1.5)$ \\
$P$-value & 0.11 & 0.22 \\
C4 (-) & $93.3(84.8-100.0)$ & $0.6(0.0-1.5)$ \\
C4 (+) & $95.3(90.7-104.8)$ & $0.6(0.0-2.4)$ \\
$P$-value & 0.17 & 0.56 \\
\hline
\end{tabular}

Rate of renal function decline (linear regression)

\begin{tabular}{cc}
\hline $\begin{array}{c}\text { Univariate }(\mathrm{ml} / \mathrm{min} \\
\left.\text { per } 1.73 \mathrm{~m}^{2} \text { per year }\right)\end{array}$ & $\begin{array}{c}\text { Mutivariate } \beta \\
(\text { s.d. })^{\mathrm{a}}\end{array}$ \\
\hline $0.4(0.1-1.2)$ & \\
$0.5(0.2-1.1)$ & $-0.01(0.40)$ \\
0.14 & 0.90 \\
$0.5(0.2-1.1)$ & \\
$0.6(0.2-1.6)$ & $-0.004(0.82)$ \\
0.22 & 0.92 \\
$0.4(0.2-0.9)$ & \\
$0.5(0.2-1.2)$ & $-0.02(0.51)$ \\
0.45 & 0.65 \\
$0.5(0.2-1.1)$ & \\
$0.3(0.1-1.4)$ & $-0.01(0.90)$ \\
0.50 & 0.72
\end{tabular}

Abbreviations: C1q (- ), absence of C1q deposits; C1q (+), presence of C1q deposits; C3 ( - ), absence of C3 deposits; C3 (+), presence of C3 deposits; C4 (-), absence of C4 deposits; C4 (+), presence of C4 deposits; IgM (-), absence of IgM deposits; IgM (+), presence of IgM deposits.

Note: Values are expressed as median (interquartile range).

Calculation of mean arterial pressure, estimated glomerular filtration rate, and proteinuria is detailed in the text.

${ }^{\mathrm{a}}$ Mutivariate models are adjusted for initial estimated glomerular filtration rate, mean arterial pressure, and proteinuria. 
Table 4 Incidence of $50 \%$ reduction in estimated glomerular filtration rate and end-stage renal disease in patients with or without glomerular IgG deposits

\begin{tabular}{|c|c|c|c|c|c|c|}
\hline & \multicolumn{4}{|c|}{ Glomerular IgG deposits } & \multirow{3}{*}{$\begin{array}{l}\text { Odds ratio with IgG } \\
\text { deposits }(95 \% \text { CI) }\end{array}$} & \multirow{3}{*}{$\mathrm{P}$-value } \\
\hline & \multicolumn{2}{|c|}{ Absence $(\mathrm{N}=427)$} & \multicolumn{2}{|c|}{ Presence $(\mathrm{N}=200)$} & & \\
\hline & $\mathrm{N}(\%)$ & $\begin{array}{l}\text { Events (1000 } \\
\text { patient-years) }\end{array}$ & $\mathrm{N}(\%)$ & $\begin{array}{l}\text { Events (1000 } \\
\text { patient-years) }\end{array}$ & & \\
\hline $\begin{array}{l}50 \% \text { reduction in estimated } \\
\text { glomerular filtration rate }\end{array}$ & $25(5.9)$ & 12.3 & $37(18.5)$ & 39.8 & $3.7(2.1-6.3)$ & $<0.001$ \\
\hline End-stage renal disease & $21(4.9)$ & 10.3 & $26(13.0)$ & 28.0 & $2.9(1.6-5.3)$ & 0.001 \\
\hline
\end{tabular}

multivariate model (model 3). Finally, glomerular IgG deposits of grade 1 (HR, 1.94; 95\% CI, 1.05-3.57; $P=0.03$ ) and grade $2+3$ (HR, 2.97; 95\% CI, 1.01-8.77; $P=0.04)$ significantly predicted adverse renal outcome independently of Oxford-MEST and clinical variables (model 4).

\section{Additive Prognostic Value of Glomerular IgG Deposition to Pathological Variables for the Prediction of Renal Outcome}

To assess the predictive power of the degree of glomerular IgG deposits, we calculated Harrell's $C$ index to be included in the multivariate Cox regression model (Table 6). Oxford-MEST lesions are significant risk factors for renal progression and the $C$-statistic of model 2 , in which the degree of glomerular IgG deposits was added, was significantly increased (C-statistic, 0.86; 95\% CI: 0.82-0.91; $P=0.022)$.

\section{Discussion}

This study showed that glomerular IgG deposition was associated with deterioration of renal function in patients with IgA nephropathy independently of proteinuria and unfavorable histopathological features, such as glomerular sclerosis or tubulointerstitial fibrosis. Moreover, adding the glomerular IgG deposition parameter improved the predictive power of the model using the Oxford-MEST score.

It is well known that under-galactosylation of O-linked carbohydrates in the hinge region of IgA1 molecules has a key role in the pathogenesis of IgA nephropathy. IgA1 with aberrant hinge region glycans or IgA1 with antiglycan specificity binds to IgG and forms circulating or in situ immune complexes. $^{8}$ Novak et $a l^{18}$ showed that immune complexes containing aberrantly glycosylated IgA1 bind to mesangial cells more efficiently than uncomplexed IgA. Eventually, subsequent deposition of immune complexes within the mesangium induces mesangial damage triggering kidney injury. ${ }^{8}$ Therefore, It could be presumed that the intensity of immune complexes composed of aberrantly glycosylated IgA1 with antigylcan-specific IgG antibody may determine the presence or absence of mesangial IgG deposition. However, concomitant IgG was variably deposited from approximately 15-80\% in patients with IgA nephropathy. ${ }^{19}$ Of note, Glassock $^{20}$ suggested that IgG antibodies to aberrantly glycosylated IgA1 were not crucial to disease pathogenesis, while mesangial IgG deposition correlated with the severity of the disease. An IgA nephropathy animal model in which mesangial IgA and IgG co-deposition aggravated renal inflammation supported this hypothesis. ${ }^{11}$ Recent observational studies have also indicated that the presence of mesangial IgG deposition had clinical significance in IgA nephropathy patients. ${ }^{13,14}$ The present study clearly demonstrated that IgA nephropathy patients with glomerular IgG deposition had poorer long-term outcomes and the inclusion of glomerular IgG deposition evaluation in IgA nephropathy provided additional prognostic value in the adjusted model.

The Oxford classification of IgA nephropathy identified four types of lesions as specific pathological features that are considered independent predictors of clinical outcomes. ${ }^{15,16}$ However, this classification does not include immunostaining patterns in the analysis. There have been several studies showing that immunofluorescent findings are related to histological severity. Bellur et al showed an association between IgG deposition and the presence of endocapillary proliferation and a higher mesangial cellularity score. Interestingly, Rifai $^{21}$ suggested that immune complexes composed of aberrantly glycosylated IgA1 with antigylcanspecific IgG antibodies caused marked macrophage activation, severe glomerular injury, and finally chronic changes, such as tuft adhesion and glomerular sclerosis. In addition, Komatsu et $a l^{22}$ reported that mesangial C3 deposition was related to $\operatorname{IgG}$ co-deposition and severe histological lesions, such as glomerular sclerosis, crescents, and tuft adhesions. Moreover, IgG deposition has also been reported to mediate renal complement activation. ${ }^{23}$ Furthermore, co-deposition of IgA with IgG caused mesangial cell proliferation, mesangiolysis, and subsequent renal injury through complement activation and fixation. ${ }^{11,23}$ These could be related to 


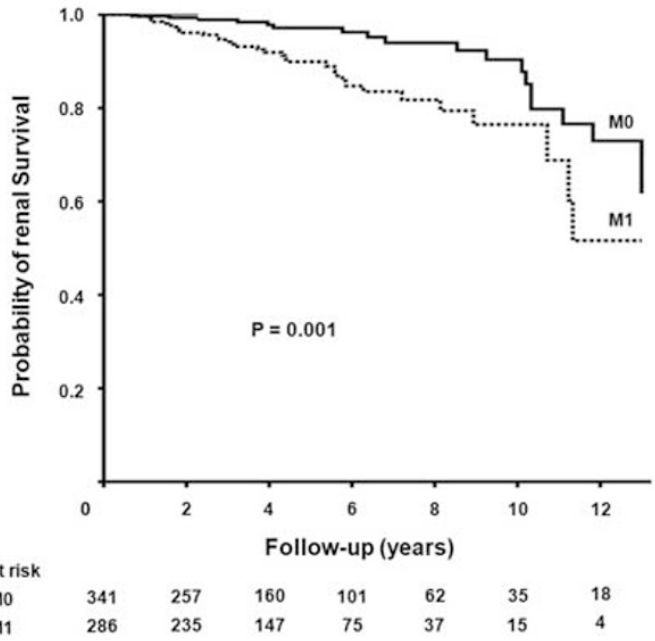

C

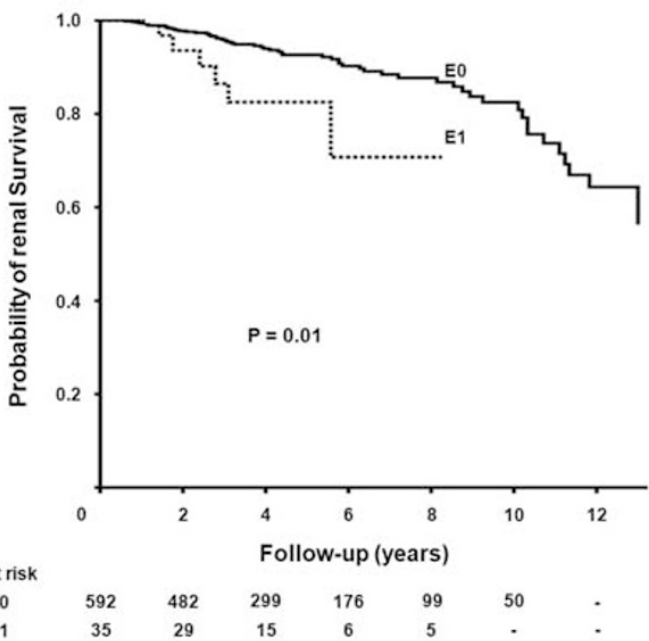

b

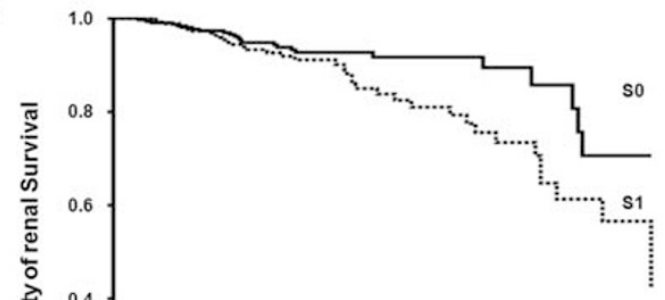

0.2

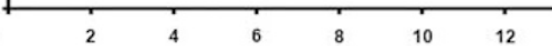

No at risk Follow-up (years)

$\begin{array}{llllllll}\text { So } & 339 & 275 & 185 & 102 & 51 & 24 & 11\end{array}$

d

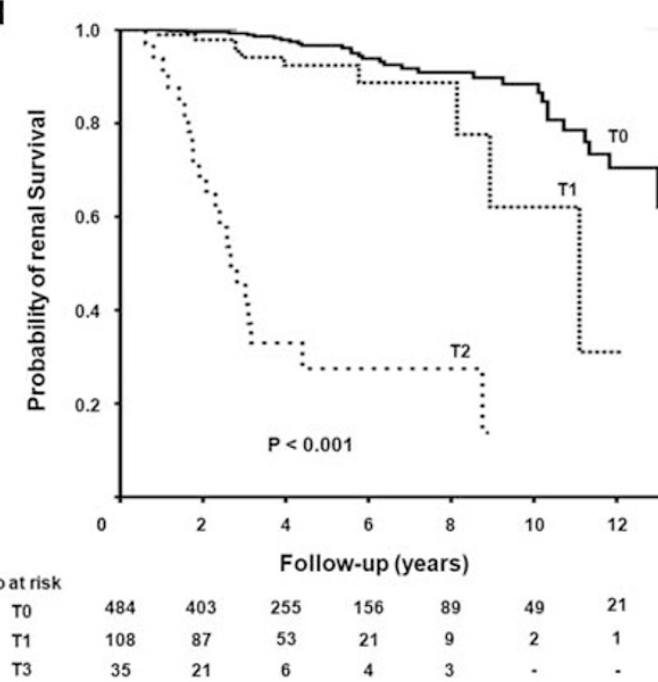

e

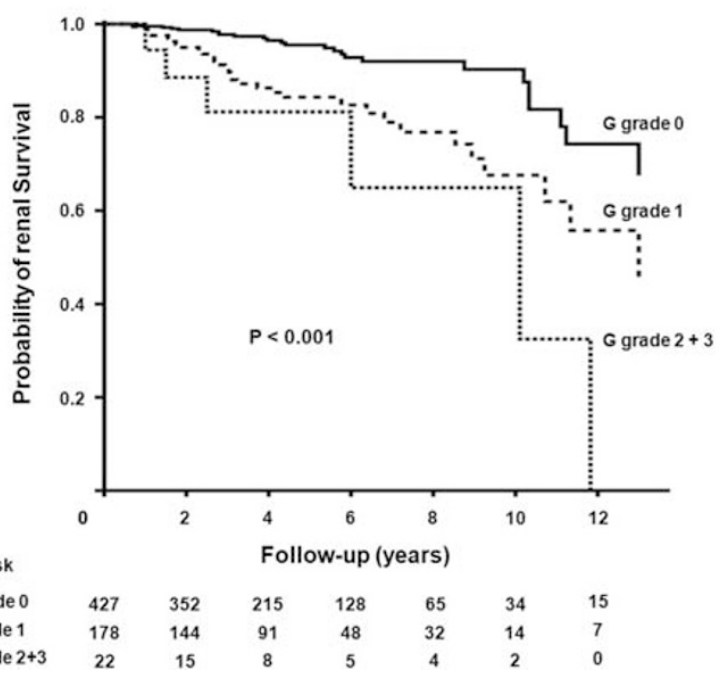

Figure 1 Kaplan-Meier analysis of cumulative renal survival of patients with IgA nephropathy according to histological variables of the Oxford classification and mesangial IgG deposits. (a) mesangial hypercellularity, (b) endocapillary proliferations, (c) segmental glomerulosclerosis, (d) tubular atrophy/interstitial fibrosis, and (e) glomerular IgG deposits (all $P<0.05$ ). 
Table 5 Multivariate Cox regression models for renal outcome of end-stage renal disease or 50\% reduction in estimated glomerular filtration rate in study subjects

\begin{tabular}{|c|c|c|c|c|c|c|c|c|}
\hline & \multicolumn{2}{|l|}{ Model $1^{\mathrm{a}}$} & \multicolumn{2}{|l|}{ Model $2^{\mathrm{b}}$} & \multicolumn{2}{|l|}{ Model $3^{\mathrm{C}}$} & \multicolumn{2}{|l|}{ Model $4^{\mathrm{d}}$} \\
\hline & HR (99\% CI) & P-value & HR (99\% CI) & P-value & HR (99\% CI) & P-value & HR (99\% CI) & $\mathrm{P}$-value \\
\hline $\begin{array}{l}\text { Mean arterial pressure (per } \\
1 \mathrm{~mm} \mathrm{Hg} \text { ) }\end{array}$ & $1.00(0.98-1.02)$ & 0.9 & $1.00(0.98-1.02)$ & 0.9 & $1.01(0.99-1.02)$ & 0.58 & $1.01(0.99-1.03)$ & 0.46 \\
\hline Proteinuria (per 1 g) & $1.47(1.29-1.67)$ & $<0.001$ & $1.39(1.21-1.60)$ & $<0.001$ & $1.24(1.04-1.47)$ & 0.01 & $1.20(1.01-1.43)$ & 0.04 \\
\hline $\begin{array}{l}\text { Estimated glomerular filtration } \\
\text { rate (per } 1 \mathrm{ml} / \mathrm{min} / 1.73 \mathrm{~m}^{2} \text { ) }\end{array}$ & $0.97(0.96-0.98)$ & $<0.001$ & $0.97(0.96-0.98)$ & $<0.001$ & $0.99(0.97-1.00)$ & 0.06 & $0.99(0.97-1.00)$ & 0.05 \\
\hline M1 (vs Mo) & & & & & $1.67(0.85-3.28)$ & 0.14 & $1.59(0.81-3.14)$ & 0.18 \\
\hline $\mathrm{S} 1$ (vs S0) & & & & & $2.73(1.49-5.03)$ & 0.001 & $2.70(1.46-5.01)$ & 0.002 \\
\hline E1 (vs E0) & & & & & $1.59(0.59-4.33)$ & 0.36 & $1.54(0.56-4.26)$ & 0.40 \\
\hline T1 (vs T0) & & & & & $2.33(1.01-5.40)$ & 0.05 & $2.18(0.95-5.03)$ & 0.07 \\
\hline $\mathrm{T} 2(v s \mathrm{~T} 0)$ & & & & & $17.39(7.12-42.45)$ & $<0.001$ & $14.94(5.99-37.26)$ & $<0.001$ \\
\hline G grade 1 (vs $\mathrm{G}$ grade 0 ) & & & $2.56(1.48-4.41)$ & 0.001 & & & $1.94(1.05-3.57)$ & 0.03 \\
\hline $\mathrm{G}$ grade $2+3($ vs $\mathrm{G}$ grade 0 ) & & & $4.31(1.61-11.5)$ & 0.004 & & & $2.97(1.01-8.77)$ & 0.04 \\
\hline
\end{tabular}

Abbreviations: CI, confidence interval; E1, presence of endocapillary hypercellularity; E0, absence of endocapillary; G grade 0, no or trace IgG deposits; G grade 1, mild IgG deposits; G grade 2, moderate IgG deposits; G grade 3, marked IgG deposits; HR, hazard ratio; M0, mesangial hypercellularity score of $\leq 0.5$; M1, mesangial hypercellularity score $>0.5$; S0, absence of segmental glomerulosclerosis; S1, presence of segmental glomerulosclerosis; T0, tubular atrophy/interstitial fibrosis $\leq 25 \%$ of cortical area; T1, tubular atrophy/interstitial fibrosis $26-50 \%$ of cortical area; T2, tubular atrophy/interstitial fibrosis $>50 \%$ of cortical area.

Calculation of mean arterial pressure, estimated glomerular filtration rate, and proteinuria is detailed in the text.

${ }^{\mathrm{a}}$ Model 1: sex, age, and baseline clinical variables (mean arterial pressure, estimated glomerular filtration rate, and proteinuria).

bodel 2: model 1+the degree of glomerular IgG deposits.

CModel 3: model 1+M, S, E, T.

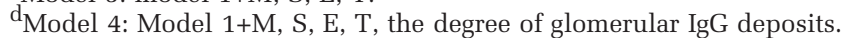

Table $6 C$-statistics for prediction of the renal outcome of endstage renal disease and $50 \%$ reduction in estimated glomerular filtration rate using multivariate Cox's regression models

\begin{tabular}{|c|c|c|}
\hline Model & C-statistics (95\% CI) & $\begin{array}{c}\text { P for difference of C-statistics } \\
\text { compared with models }\end{array}$ \\
\hline Model $1^{\mathrm{a}}$ & $0.84(0.79-0.90)$ & 0.02 \\
\hline Model $2^{\mathrm{b}}$ & $0.86(0.82-0.91)$ & \\
\hline
\end{tabular}

Abbreviation: CI, confidence interval.

${ }^{a}$ Model 1: Oxford-MEST.

bodel 2: Oxford-MEST+the degree of glomerular IgG deposits (G0, G1, and G2+3).

the greater potential of IgG to initiate and sustain an inflammatory process, thus interfering with the paracrine and endocrine functions of the mesangium. It is known that this local complement system in the glomeruli is activated via the alternative or mannose-binding lectin pathway in IgA nephropathy. ${ }^{24,25}$ Complement by IgG antibodies proceeds through the classic pathway, as demonstrated by $\mathrm{C} 1$ binding. ${ }^{26}$ In other words, IgG forms the classic pathway convertase C4bC2a through C1 fixation and subsequently cleaves C3 into C3a and C3b. A greater number of complement-activating moieties in the mesangial area result in more C3 convertase formation, including the alternative pathway convertase $\mathrm{C} 3 \mathrm{bBb}$. The alternative pathway C3 convertase subsequently cleaves C3 and generate more C3 cleavage products. ${ }^{11}$ Therefore, we hypothesize that classic complement pathway by IgG in $\operatorname{Ig} \mathrm{A}$ nephropathy may induce prolong and more severe type of glomerulonephritis. In line with this theory, it was observed that the intensity of glomerular IgG depo-

sits had strong positive correlation with the intensity of C3 deposit in this study (data not shown), and glomerular IgG deposition was significantly associated with tubulo-interstitial fibrosis as an indicator of advanced renal pathology in this study. In addition, this present study also showed that baseline urinary protein-to-creatinine ratio is significantly correlated with glomerular IgG deposition.

Of note, the present study did not show the independent association of renal outcomes with $\mathrm{M}$ or $\mathrm{E}$ lesion discrepant from previous validation studies of Oxford classification. ${ }^{15,27,28}$ These observations might be partly arisen from the relatively low proportion of E1 lesion and the small number of corticosteroid users compared with previous studies. Most of the patients were treated with ACEi or ARBs during the follow-up regardless of pathological grade. Main decisions used to guide treatment options are largely based on clinical features such as the amount of proteinuria, not pathological features. Additionally, corticosteroid treatment bias was observed for E lesion, whereas there was no such association for $\mathrm{M}$ and $\mathrm{S}$ lesions (data not shown). Furthermore, a small number of patients with E1 lesion could give rise to no correlation between glomerular IgG deposition and endocapillary hypercellularity scores. 
Our study had several limitations. First, this was a retrospective study, thus its observational nature limits our findings, suggesting that glomerular IgG deposition in patients with IgA nephropathy actually contributes to the progression of IgA nephropathy. Additionally, there are potential confounding factors affecting renal progression. However, we should underline that there was no significant difference in the time to renal biopsy between IgA nephropathy patients with glomerular IgG deposition compared with those without glomerular IgG deposition. Furthermore, because the method of immunofluorescence was basically same throughout the research period, there were no differences in methodology. Second, the numbers of patients with moderate and marked glomerular IgG deposits were small. Although the groups of moderate glomerular IgG deposits and marked glomerular IgG deposits was combined and then we verified the correlation of renal outcome with intensity of glomerular IgG positivity, more patients with moderate glomerular IgG deposits and marked glomerular IgG deposits are needed to validate these findings. Third, we did not measure serum antigylcan-specific IgG antibodies to aberrantly glycosylated IgA1. Although there were no differences in serum IgG concentrations according to the degree of glomerular IgG deposits, it is unclear whether the presence of mesangial IgG deposition in a fraction of patients with IgA nephropathy could reflect fluctuations of specific IgG autoantibody levels in the present study. Suzuki et $a l^{9}$ reported that serum antigylcan-specific $\operatorname{IgG}$ antibodies to aberrantly glycosylated IgA1 are associated with the development of IgA nephropathy and might represent a disease-specific marker and potential therapeutic target. Therefore, measurement of serum antigylcan-specific IgG antibodies would be helpful to further clarify the clinical implications of mesangial IgG deposition in patients with IgA nephropathy. Finally, we did not routinely perform IgG subtyping at the time of the diagnosis. So, we retrieved additional snap-frozen sectioned slides and performed immunofluorescent microscopy for IgG1, IgG2, IgG3, and IgG4 in a small number of cases. However, most of the results were not reliable owing to disappearance of antigenicity during the storage period. To evaluate the significance of IgG subtype in IgA nephropathy with IgG deposits, prospective study or retrospective study using archival paraffinembedded tissue is required.

In conclusion, this study showed that glomerular IgG deposits were independently associated with poor renal outcome in patients with IgA nephropathy. In addition, glomerular IgG deposition correlated with greater histological activity and increased clinical severity. Thus, these findings suggest that glomerular IgG deposition may have a role in the progression of IgA nephropathy. Our findings require validation in other cohorts.

\section{Acknowledgments}

This work was supported by the Brain Korea 21 PLUS Project for Medical Science, Yonsei University College of Medicine and a grant of the Korea Healthcare Technology R\&D Project, Ministry of Health and Welfare, Republic of Korea (HI10C2020).

\section{Disclosure/conflict of interest}

The authors declare no conflict of interest.

\section{References}

1 D'Amico G. The commonest glomerulonephritis in the world: IgA nephropathy. Q J Med 1987;64:709-727.

2 Bogenschutz O, Bohle A, Batz C et al. IgA nephritis: on the importance of morphological and clinical parameters in the long-term prognosis of 239 patients. Am J Nephrol 1990;10:137-147.

3 Johnston PA, Brown JS, Braumholtz DA et al. Clinicopathological correlations and long-term follow-up of 253 United Kingdom patients with IgA nephropathy. A report from the MRC Glomerulonephritis Registry. Q J Med 1992;84:619-627.

4 Radford MG Jr., Donadio JV Jr., Bergstralh EJ et al. Predicting renal outcome in IgA nephropathy. J Am Soc Nephrol 1997;8:199-207.

5 D'Amico G. Natural history of idiopathic IgA nephropathy: role of clinical and histological prognostic factors. Am J Kidney Dis 2000;36:227-237.

6 D'Amico G. Natural history of idiopathic IgA nephropathy and factors predictive of disease outcome. Semin Nephrol 2004;24:179-196.

7 Reich HN, Troyanov S, Scholey JW et al. Remission of proteinuria improves prognosis in IgA nephropathy. J Am Soc Nephrol 2007;18:3177-3183.

8 Floege J. The pathogenesis of IgA nephropathy: what is new and how does it change therapeutic approaches? Am J Kidney Dis 2011;58:992-1004.

9 Suzuki H, Fan R, Zhang Z et al. Aberrantly glycosylated IgA1 in IgA nephropathy patients is recognized by IgG antibodies with restricted heterogeneity. J Clin Invest 2009;119:1668-1677.

10 Rodicio JL. Idiopathic IgA nephropathy. Kidney Int 1984;25:717-729.

11 van Dixhoorn MG, Sato T, Muizert Y et al. Combined glomerular deposition of polymeric rat IgA and IgG aggravates renal inflammation. Kidney Int 2000;58: 90-99.

12 Nieuwhof C, Kruytzer M, Frederiks P et al. Chronicity index and mesangial IgG deposition are risk factors for hypertension and renal failure in early IgA nephropathy. Am J Kidney Dis 1998;31:962-970.

13 Wada Y, Ogata H, Takeshige Y et al. Clinical significance of IgG deposition in the glomerular mesangial area in patients with IgA nephropathy. Clin Exp Nephrol 2013;17:73-82.

14 Bellur SS, Troyanov S, Cook HT et al. Immunostaining findings in IgA nephropathy: correlation with histology and clinical outcome in the Oxford classification patient cohort. Nephrol Dial Transplant 2011;26: 2533-2536. 
15 Cattran DC, Coppo R, Cook HT et al. The Oxford classification of IgA nephropathy: rationale, clinicopathological correlations, and classification. Kidney Int 2009;76:534-545.

16 Roberts IS, Cook HT, Troyanov S et al. The Oxford classification of IgA nephropathy: pathology definitions, correlations, and reproducibility. Kidney Int 2009;76:546-556.

17 Levey AS, Stevens LA, Schmid CH et al. A new equation to estimate glomerular filtration rate. Ann Intern Med 2009;150:604-612.

18 Novak J, Tomana M, Matousovic K et al. IgA1containing immune complexes in IgA nephropathy differentially affect proliferation of mesangial cells. Kidney Int 2005;67:504-513.

19 Habib R, Niaudet P, Levy M. Schonlein-Henoch purpura nephritis and IgA nephropathy. In: Tisher CC, Brenner BM (eds). In: Renal Pathology with Clinical and Functional Correlations. J.B. Lippincott \& Co.: Philadelphia, Pennsylvania, USA, 1994, pp 472-523.

20 Glassock RJ. Analyzing antibody activity in $\operatorname{IgA}$ nephropathy. J Clin Invest 2009;119:1450-1452.

21 Rifai A. IgA nephropathy: immune mechanisms beyond IgA mesangial deposition. Kidney Int 2007;72:239-241.
22 Komatsu H, Fujimoto S, Hara S et al. Relationship between serum IgA/C3 ratio and progression of IgA nephropathy. Intern Med 2004;43:1023-1028.

23 Waldo FB. Role of IgA in IgA nephropathy. J Pediatr 1990;116:S78-S85.

24 Endo M, Ohi H, Ohsawa I et al. Glomerular deposition of mannose-binding lectin (MBL) indicates a novel mechanism of complement activation in IgA nephropathy. Nephrol Dial Transplant 1998;13: 1984-1990.

25 Nakagawa H, Suzuki S, Haneda M et al. Significance of glomerular deposition of C3c and C3d in IgA nephropathy. Am J Nephrol 2000;20:122-128.

26 Medgyesi GA, Fust G, Gergely J et al. Classes and subclasses of rat immunoglobulins: interaction with the complement system and with staphylococcal protein A. Immunochemistry 1978;15:125-129.

27 Lee H, Yi SH, Seo MS et al. Validation of the Oxford classification of IgA nephropathy: a single-center study in Korean adults. Korean J Intern Med 2012;27:293-300.

28 Zeng $\mathrm{CH}$, Le W, Ni Z et al. A multicenter application and evaluation of the oxford classification of IgA nephropathy in adult chinese patients. Am J Kidney Dis 2012;60:812-820.

Supplementary Information accompanies the paper on Modern Pathology website (http://www.nature.com/ modpathol) 